\title{
ATLAS, CMS and LHCb Trigger systems for flavour physics
}

\section{Luigi GUIDUCCI for the ATLAS, CMS and LHCb collaborations*}

Università degli Studi di Bologna and INFN

E-mail: guiducciebo.infn.it

\begin{abstract}
The trigger systems of the LHC detectors play a crucial role in determining the physics capabilities of the experiments. A reduction of several orders of magnitude of the event rate is needed to reach values compatible with detector readout, offline storage and analysis capability. The main design features of the ATLAS, CMS, and LHCb trigger systems and their operation during the first LHC run in 2011 and 2012 are described. Performance results in terms of rates and efficiencies of the trigger systems will be shown, with an emphasis on aspects related to flavour physics.
\end{abstract}

14th International Conference on B-Physics at Hadron Machines, April 8-12, 2013

Bologna, Italy

\footnotetext{
* Speaker.
} 


\section{Introduction}

The trigger and data acquisition systems of LHC detectors have a crucial role, as they implement a reduction of the event rate of several orders of magnitude, from the very high interaction rate provided by the LHC accelerator to the storage of interesting events. Many requirements constrain the design of a trigger system, including the detector and the data acquisition system design and performance, the experimental physics program, the instantaneous luminosity provided by the accelerator, the data storage and processing capabilities, and the technologies available at the moment of the construction.

In the following the main design and performance figures of the trigger systems of the ATLAS, CMS, and LHCb detectors are described and compared.

\section{ATLAS, CMS and LHCb: detectors and data taking conditions}

The LHCb [1] detector is a forward spectrometer, covering the region $2<\eta<5$. Close to the interaction point (IP), a silicon strip tracking system resolves the primary and secondary vertices. The tracking is complemented with stations of silicon strips and straw tubes, and by RICH detectors for particle identification. Further away from the IP there are the electromagnetic and hadronic calorimeters, followed by muon detectors for energy measurements and particle identification. They are the only detectors used as input to the first level of the trigger system.

THe ATLAS [2] and CMS [3] general purpose detectors have a barrel-endcap geometry and almost $4 \pi$ acceptance. They rely on large volume, high field superconducting solenoids (and toroids in case of ATLAS) to achieve good track separation in high pileup events and enable fine grained, $\sim 100$ million channels tracking systems to achieve good transverse momentum $\left(p_{\mathrm{T}}\right)$ resolution up to and beyond $1 \mathrm{TeV} / \mathrm{c}$. Hermetic calorimetry and muon chambers complete the detector designs.

During 2012, the LHC operated at a bunch spacing of $50 \mathrm{~ns}$ with small $\beta^{*}=0.6 \mathrm{~m}$ at the ATLAS and CMS interaction regions. The peak luminosity in the ATLAS and CMS interaction points routinely reached $\sim 7.5 \times 10^{33} \mathrm{~cm}^{-2} \mathrm{~s}^{-1}$ (see Fig. 1). The peak pileup reached $\sim 35$ interactions per crossing, well beyond the pileup value foreseen by the LHC design of about 20 interactions per crossing. The high pileup environment presented challenges for the detector and event reconstruction, in particular in two aspects. First, there is a high hit multiplicity in the inner detectors, resulting in larger event size and more complicated tracking. Second, due to the energy pileup in the calorimeters, the calorimeter-based first level trigger, where tracking information is not used and it is not possible to distinguish the different primary interactions, is a very challenging task.

LHCb is optimized for b-physics, which has a very large cross section at the LHC, and was thus designed to operate at leveled luminosity, to limit the pileup and obtain the cleanest reconstruction of B decays. The instantaneous luminosity in 2012 was normally $\sim 4 \times 10^{32} \mathrm{~cm}^{-2} \mathrm{~s}^{-1}$, larger than the design value and corresponding to an average pileup of $\sim 1.7$ interactions per crossing.

\section{Trigger architectures}

A schematic representation of the trigger architectures is shown in Fig. 2. A common constraint is the maximum readout rate from the detector, usually one or two orders of magnitude 

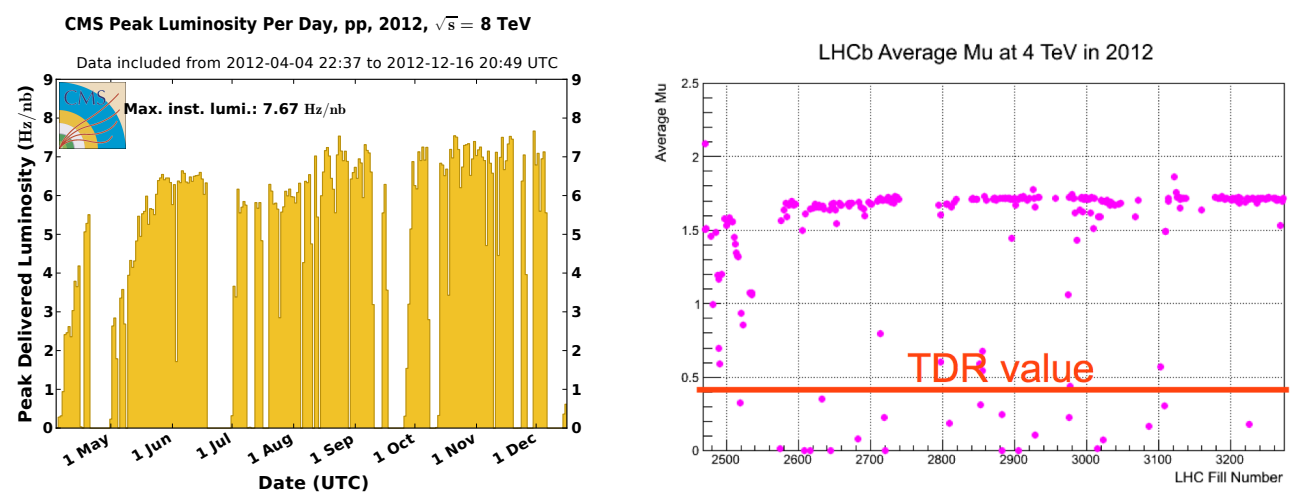

Figure 1: Left: peak luminosity per day as measured in the CMS detector in 2012. Values up to and above $\sim 7.5 \times 10^{33} \mathrm{~cm}^{-2} \mathrm{~s}^{-1}$, corresponding to $\sim 35$ interactions per crossing, were reached in 2012. Right: average number of interactions per crossing (mu) in the LHCb detector, during part of the 2012 data taking. Values more than three times larger than foreseen at the design stages ("TDR value" in the plot) were routine.

smaller than the $\mathrm{p}-\mathrm{p}$ interaction rate. Thus, all three trigger systems considered have an initial trigger (called Level-1 in ATLAS and CMS and Level-0 in LHCb) which is based on custom-built hardware, running at $40 \mathrm{MHz}$ in a pipelined architecture, providing a fixed-latency and dead-time free decision about the bunch crossings to readout. The first-level trigger systems are based on coarse-grained position and energy information from the calorimeters and muon detectors. The maximum rate of events accepted by the first-level trigger is about $1 \mathrm{MHz}$ in $\mathrm{LHCb}$ and about $100 \mathrm{kHz}$ in ATLAS and CMS.

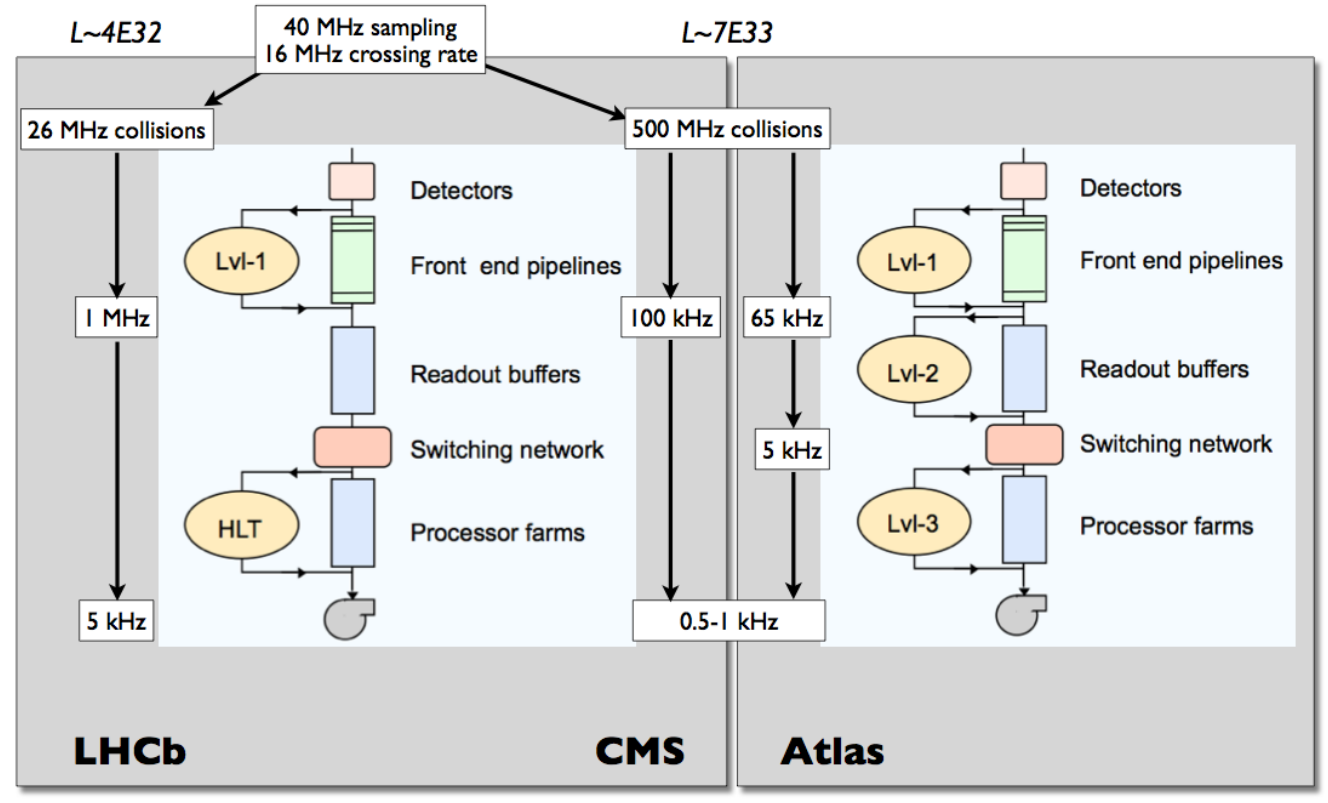

Figure 2: Schematic structures of the LHCb, ATLAS and CMS trigger systems. Typical luminosity during 2012 data taking and event rates at different stages are quoted. 
Full granularity readout data is available to the subsequent high level trigger (HLT) stage to reduce the rate further. ATLAS and CMS ran in 2012 with an average rate to storage in the range $500-1000 \mathrm{~Hz}$, while LHCb reached about $5 \mathrm{kHz}$. In case of ATLAS, the HLT is split into two levels, called Level-2 (L2) and Event Filter (EF). The L2 system acts on data from a limited Region of Interest, as defined by the Level-1 system, effectively reducing the input bandwidth, simplifying the event building and allowing sophisticated algorithms to run in shorter time. If the refined measurement of the trigger object obtained by the L2 algorithms pass the trigger threshold and requirements, the full detector event building is performed and the final (EF) algorithms are executed. CMS and LHCb have a single stage HLT: the full detector is read out and full event building is performed immediately. Nevertheless the algorithms are designed in several subsequent stages, progressively more complex - as they must be executed on a smaller number of events surviving the previous selections - and the Region of Interest concept is implemented in the software architecture.

The LHCb trigger system is designed to select B decays into charged tracks, most interesting charm meson decays, prompt dimuon events, while keeping high efficiency for rare B decays (photons, muons).

The main goals of the ATLAS and CMS trigger systems are to keep a high trigger efficiency for electroweak and new physics while reducing the QCD background, using reconstruction of high transverse momentum electrons and photons, muons, jets, and missing energy. The B-physics program in ATLAS and CMS is essentially limited to decays with dimuons in the final state, as it would be nearly impossible to select heavy flavour hadronic decays from the high QCD background.

\section{The first level trigger}

In $\mathrm{LHCb}$, the hadron/photon/electron candidates are built by summation of energy into projective towers of the electromagnetic and hadronic calorimeters and applying a threshold on the highest energy tower. Muon candidates are built with pattern-finding processors, which also assign a transverse momentum with $\sim 25 \%$ resolution using hits from pads and strips of the muon chambers. Thresholds on the largest (and second largest) transverse momentum are applied for muon (dimuon) triggers. Typical thresholds on transverse momentum/energy are 1.5, 2.5, and $3.5 \mathrm{GeV}$ for muon, electron/photon, and hadron triggers, respectively. The efficiency for the muon/dimuon and electron trigger as a function of the object transverse momentum/energy are shown in Fig. 3 [4].

The ATLAS [5] and CMS [6] Level-1 triggers are also based on calorimeter and muon information. In CMS all three muon detectors [7] (RPC and DT in the barrel and RPC and CSC in the endcaps) are used to reconstruct Level-1 muons independently and a Global Muon Trigger stage has the task of merging the subsystem candidates. The muon trigger based on RPC chambers searches for muon candidates by comparing the hits from the detector to a large number of predefined patterns determined from simulation, providing also a transverse momentum estimation. Independently, DT and CSC data are used to build track segments from individual muon chambers, then a track finding algorithm based on extrapolation and matching to subsequent muon stations is used to generate muon track candidates. The transverse momentum is estimated from the track bending in the return yoke field, by using the position and angle of the track segments used to 

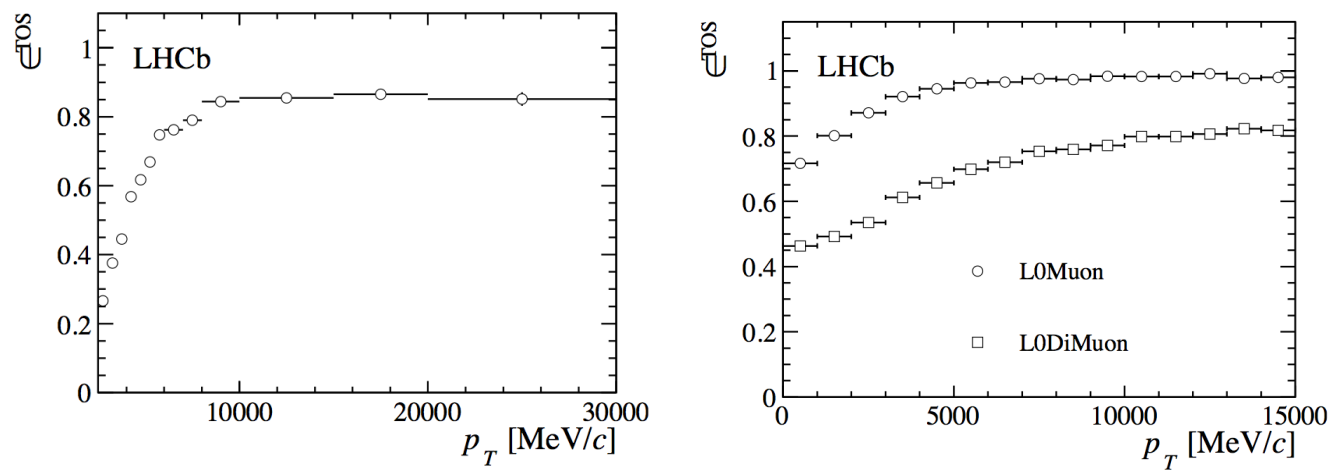

Figure 3: Left: efficiency of the LHCb L0 single electron trigger, evaluated in events containing the B decay $\mathrm{B}^{0} \rightarrow \mathrm{J} / \psi\left(\mathrm{e}^{+} \mathrm{e}^{-}\right) \mathrm{K}^{* 0}$, as a function of the $\mathrm{J} / \psi$ transverse momentum. Right: efficiency of the LHCb L0 muon trigger, evaluated in events containing the $\mathrm{B}$ decay $\mathrm{B}^{+} \rightarrow \mathrm{J} / \psi \mathrm{K}^{+}\left(\mathrm{J} / \psi \rightarrow \mu^{+} \mu^{-}\right)$as a function of the $\mathrm{J} / \psi$ transverse momentum.

build the muon. A global processor merges the candidates produced independently from the three subdetector processors.

The Level-1 trigger in ATLAS has a single muon processor, using RPC and TGC detectors in barrel and endcap, respectively, while another design feature is the connection to the L2-trigger to provide the Region of Interest for the subsequent processing. The Level-1 muon trigger is based on coincidences between different layers of muon trigger chambers. There are three layers of RPC (barrel) or TGC (endcap) covering the muon system acceptance [8]. The intermediate layer defines a reference point; the width of the window where hits are accepted for coincidences in the inner (outer) layer defines the transverse momentum cut applied to the muon candidate in low (high) transverse momentum categories.

As an example, the efficiency for Level-1 single muon trigger with $p_{\mathrm{T}}$ threshold at $11 \mathrm{GeV}$ in the forward region of ATLAS is shown in Fig. 4 (left). Figure 4 (right) shows the efficiency for the CMS Level-1 muon trigger with a $p_{\mathrm{T}}$ threshold at $14 \mathrm{GeV}$; the two sets of points indicate 2011 data (red) and 2012 data (blue). The slightly lower efficiency in 2012 results from tightened quality cuts which allowed the trigger rate to be reduced by about $50 \%$ [9].

Trigger algorithms are combined in a so-called Trigger Menu. As an example, in Fig. 5, left, typical thresholds and rates for the main ${ }^{1}$ CMS Level-1 trigger algorithms used during 2012 data taking are shown; the ATLAS numbers are not very different. The main Level-1 trigger algorithm for beauty and charm physics is a double muon trigger, limited to $|\eta|<2.1$, with a very low cut in transverse momentum ${ }^{2}$.

In Fig. 5, right, the total Level-1 rate, as recorded by CMS during a real LHC fill, is shown. Initial instantaneous luminosity was about $\sim 7.5 \times 10^{33} \mathrm{~cm}^{-2} \mathrm{~s}^{-1}$ in this fill and the corresponding

\footnotetext{
${ }^{1}$ Inclusive single and double object triggers, and main cross triggers, summing up to most of the total CMS Level-1 rate.

${ }^{2}$ No explicit $p_{\mathrm{T}}$ cut was applied by Level-1 trigger in CMS. Due to the magnetic field geometry and the detector material, the muon identification efficiency reaches plateau at $\sim 5 \mathrm{GeV}$ at central pseudorapidity and at $\sim 2 \mathrm{GeV}$ at large pseudorapidity
} 

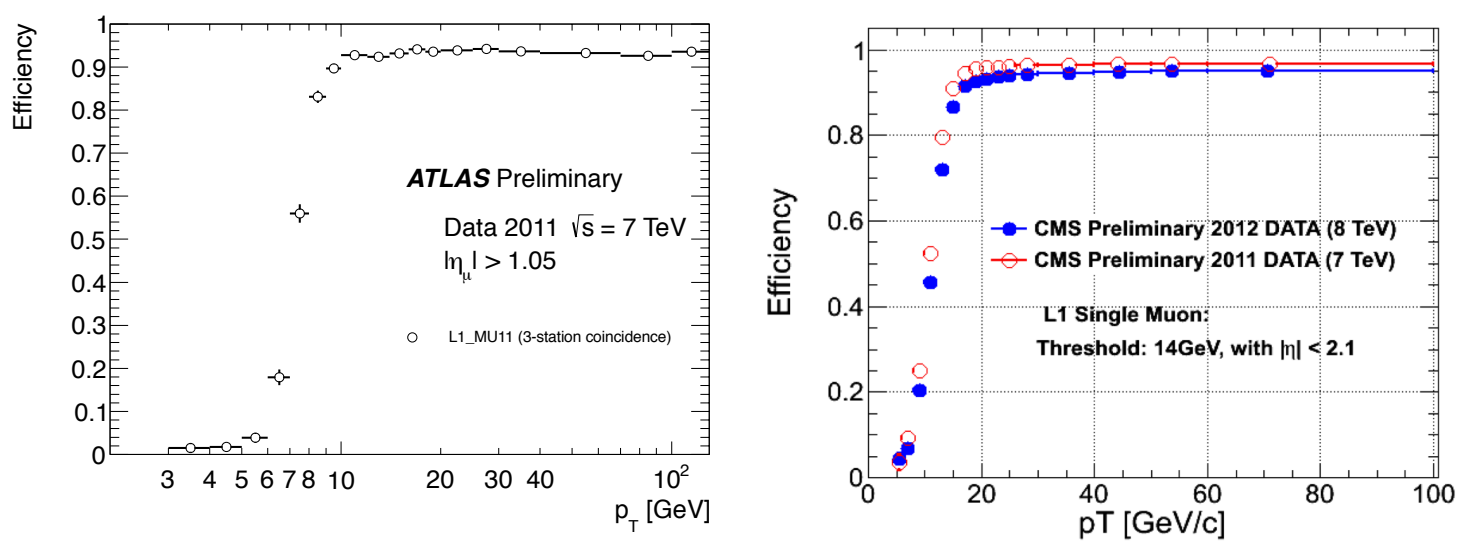

Figure 4: Left: efficiency versus $p_{\mathrm{T}}$ for ATLAS Level-1 single muon trigger with threshold at $11 \mathrm{GeV}$, in the endcap region. Right: CMS single muon trigger efficiency, for $|\eta|<2.1$ and $p_{\mathrm{T}}$ cut at $14 \mathrm{GeV}$. Both 2011 (red) and 2012 (blue) data are shown. The single muon trigger in 2012 was configured with tightened quality cuts, allowing the rate to be reduced by $\sim 50 \%$, at the cost of a small inefficiency.

Level-1 rate about $95 \mathrm{kHz}$. The data acquisition dead-time started at $\sim 5 \%$, rapidly decreasing below $\sim 3 \%$. The discontinuity in the rate corresponds to a change of prescale set, where the total rate is filled up again by means of unprescaling lower threshold physics triggers and reducing the prescales on monitoring and calibration triggers.

\section{The high level trigger in $\mathrm{LHCb}$}

The LHCb High Level Trigger is divided in two stages: HLT1 and HLT2. In HLT1, the full reconstruction in the silicon vertex locator detector is performed. Primary vertices are built and candidate tracks are selected according to reconstruction quality and their impact parameter with respect to the associated primary vertex. The main selection algorithm is called the inclusive track trigger, where a large impact parameter and a cut on the transverse momentum of individual tracks are required; the track reconstruction is completed with the inner and outer tracking detectors, using only hits in a region of interest. This selection is run on all events, regardless of the Level-0

\begin{tabular}{|c|c|c|}
\hline L1Trigger & Threshold $(\mathbf{G e V})$ & Rate at 6.5E33 (kHz) \\
\hline Single e/gamma & 20 & 13 \\
\hline Double e/gamma & 13,7 & 8 \\
\hline Single muon & $14(\mid$ eta $\mid<2.1)$ & 7 \\
\hline Double muon & 10,0 & 6 \\
\hline Double muon low Pt & $0,0(\mid$ eta $\mid<2.1)$ & 9 \\
\hline e/gamma + muon & $12,3.5$ & 3 \\
\hline muon + e/gamma & 12,7 & 1.5 \\
\hline Single Jet & 128 & 1.5 \\
\hline Quad Jet & 36 & 3.5 \\
\hline $\mathrm{H}_{\mathrm{T}}$ & 150 & 5 \\
\hline $\mathrm{E}_{\mathrm{T}}{ }^{\text {miss }}$ & 36 & 8 \\
\hline
\end{tabular}

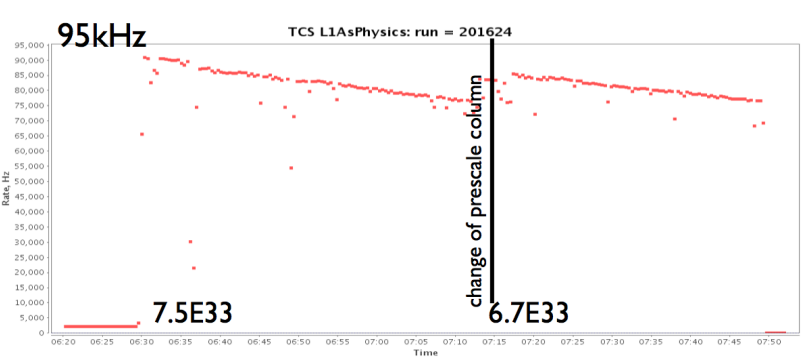

Figure 5: Left: main Level-1 Triggers in CMS with thresholds and typical rates. Right: the total CMS Level-1 trigger rate as a function of time during a typical LHC fill in 2012. 
condition. If the Level-0 condition was a muon or dimuon trigger, the track is also matched with muon detector hits in a region of interest. The muon identification allows more relaxed cuts on IP and momentum. In the case of dimuons a cut on the dimuon mass is also applied.

The subsequent stage, HLT2, is also called the topological trigger. In principle this algorithm can cover all B decays with at least two charged tracks forming a displaced vertex. First, a displaced vertex is built with a pair of selected (chi-squared, impact parameter, muon/electron ID) tracks; the distance-of-closest-approach of the two-track candidate to the primary vertex is used to choose between keeping the two-track candidate, or consider it as a seed for trying three-track vertexing. These steps are repeated, building vertices made of up to four tracks. The mass of the candidate is corrected adding the momentum transverse to the direction of flight which forces the candidate to point to the associated primary vertex and a discretized boosted decision tree is used to discriminate between signal and background [10].

The performance of the HLT2 topological trigger is illustrated in Fig. 6 for the $\mathrm{B}^{+} \rightarrow \mathrm{J} / \psi \mathrm{K}^{+}$ decays. The inclusive performance is represented by the Hlt2Topo2Body trigger which requires only two tracks from the decay products. Adding three- and four- tracks topological triggers (HLT2Toponbody) and a topological trigger with muon identification (TopoMu or Topo) increases the efficiency at low transverse momentum [4]. Exclusive triggers, dedicated to specific charm decays, are also implemented.

\section{High level muon triggers in ATLAS and CMS}

In ATLAS, the muon Level-1 trigger estimates $p_{\mathrm{T}}$ and identifies the Region Of Interest where the High Level Trigger will try to reconstruct a muon candidate and measure its transverse momentum [11]. At the Level-2, the information from Drift Tubes is used to refine the Level-1 candidate; fast fitting algorithms and look up tables are used. Then a fast combination algorithm matches a track from the Inner Detector to refine the track parameter resolution. At the Event Filter, sim-
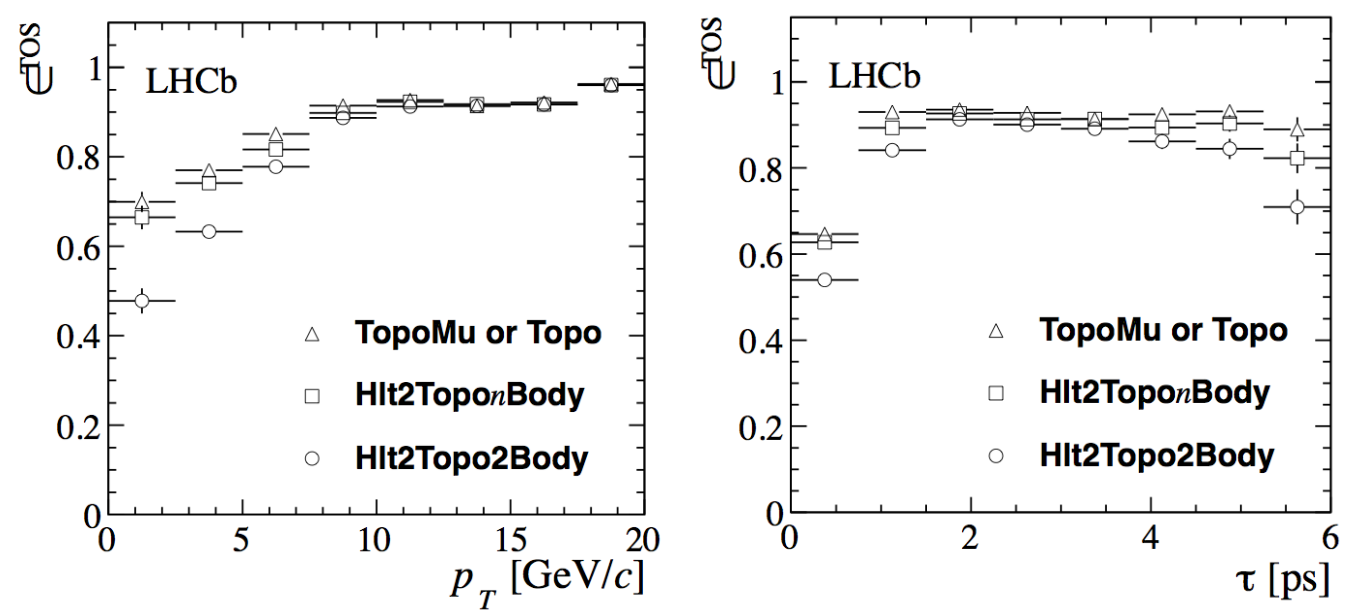

Figure 6: Efficiency of the topological triggers, evaluated on $\mathrm{B}^{+} \rightarrow \mathrm{J} / \psi \mathrm{K}^{+}$decays, as a function of $p_{\mathrm{T}}$ and $\tau$ of the $\mathrm{B}^{+}$. 

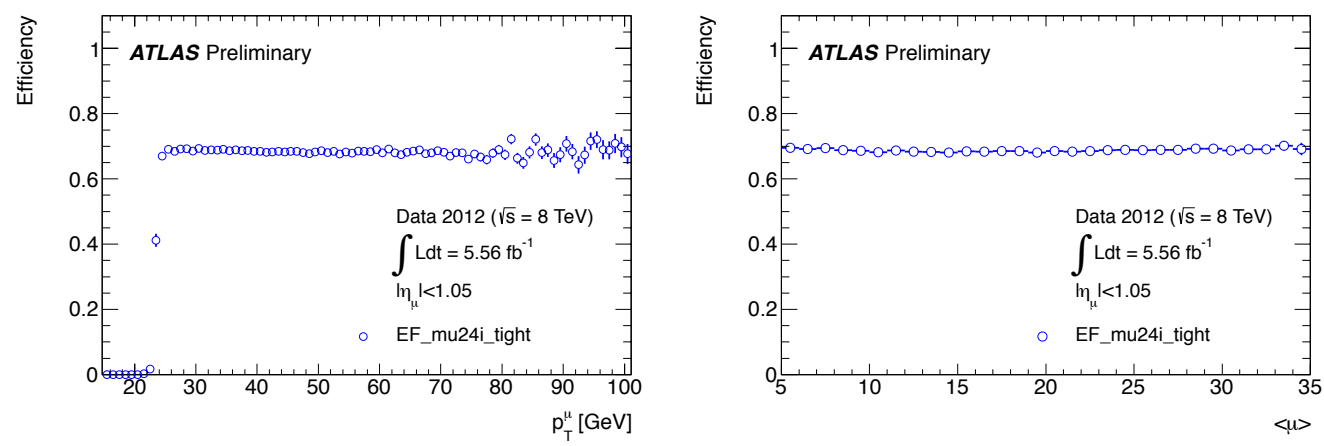

Figure 7: Left: ATLAS EF single isolated muon trigger efficiency, with $p_{\mathrm{T}}$ cut at $24 \mathrm{GeV}$, versus the $p_{\mathrm{T}}$ as measured offline. The efficiency is determined with a tag-and-probe method using muons from $\mathrm{Z}$ decays in data. Right: the same shown as a function of the average pileup, showing no dependence of the trigger efficiency from the vertex multiplicity, despite the presence of an isolation requirement.

ilar steps as performed at the L2 are executed using more precise fitting and $p_{\mathrm{T}}$ determination algorithms, derived from offline reconstruction tools. Calorimetric and track-based isolation is computed. In Fig. 7, the efficiency of the main isolated single muon trigger in ATLAS is shown, as a function of the muon transverse momentum (left) and the event pileup (right). The plots refer to the barrel region, and include geometrical acceptance effects (a region devoted to inner detectors services is not covered by muon chambers). The efficiency is determined from tag-and-probe method using $\mathrm{Z}$ boson events in data [13].

The CMS HLT [12] muon trigger algorithm is similar. First, a muon candidate is built with information from muon chambers only. If quality and transverse momentum requirements are satisfied, the algorithm extrapolates the candidate track to the inner tracking detectors. Compatible hits are used to build a combined muon candidate and the inner track momentum is used as the estimation of the muon momentum. In Fig. 8, the performance of CMS muon triggers is evaluated in terms of purity and cross-section $[14,15]$. On the left, the fraction of events satisfying progressively more stringent cuts on the offline-reconstructed muons is shown, for a sample of isolated muon triggers with $p_{\mathrm{T}}$ cut at $24 \mathrm{GeV}$. After all offline cuts, only $\sim 15 \%$ of the events are discarded. On the right of Fig. 8 the cross section of the single muon trigger with $p_{\mathrm{T}}$ cut at $40 \mathrm{GeV}$ is shown, versus the instantaneous luminosity. Both 2011 (blue) and 2012 (red) data are shown. The dependency of the cross-section on luminosity, visible in 2011 data, was removed in 2012, using optimized quality cuts on the muon candidates to remove fakes.

The high level triggers are combined in a full trigger menu. As an example, the main triggers from the ATLAS trigger menu of 2012 are reported in Fig. 9 (left); the CMS triggers are not very different.

The ATLAS and CMS muon triggers for Heavy Flavor exploit vertex (vertexing of the two muon candidates, and possibly a cut on the vertex displacement with respect to the primary vertex), mass and momentum constraints, with loose cuts on the transverse momentum of the individual muons and on the dimuons. Fig. 9 (right) shows the CMS dimuon mass spectrum, divided into contributions from different HLT paths. On top of the general, high- $p_{\mathrm{T}}$ double muon trigger, several 

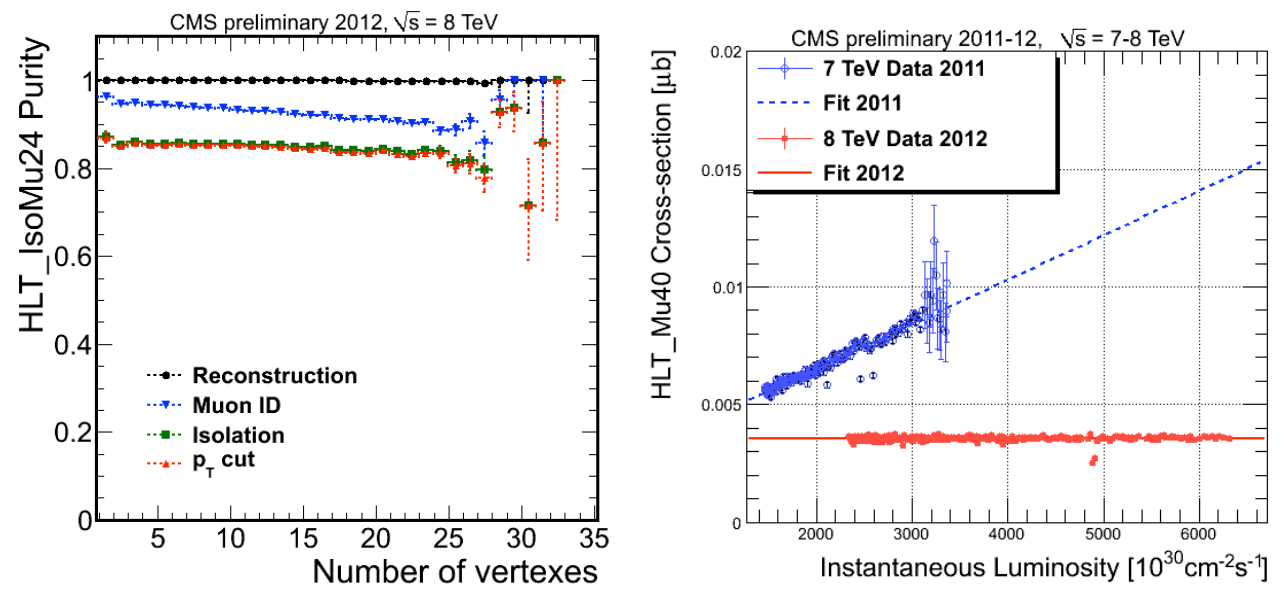

Figure 8: Left: the HLT single isolated muon trigger with $p_{\mathrm{T}}$ threshold at $24 \mathrm{GeV}$ is studied in CMS comparing the trigger object to offline reconstructed objects. Points in different colors represent the fraction of triggered events passing offline cuts: black, an offline reconstructed muon is present; blue, stringent offline quality cuts are applied; green, offline isolation cut is applied; red, offline $p_{\mathrm{T}}$ cut is applied. Right: the cross-section of CMS HLT single muon trigger with $p_{\mathrm{T}}$ threshold at $40 \mathrm{GeV}$ versus the instantaneous luminosity. A non-constant cross-section was found in 2011 data (blue points); this behavior was corrected with improved quality cuts in 2012 (red points).

triggers active within relatively small dimuon mass windows are present, and increase the collected statistics in the regions of interesting signals, such as quarkonia resonances and rare decays of $\mathrm{B}$ hadrons.

\begin{tabular}{|c|c|c|}
\hline EF Trigger & Threshold $(\mathbf{G e V})$ & Rate at 5E33 (Hz) \\
\hline Inclusive electron & 25 & 70 \\
\hline Inclusive muon & 24 & 45 \\
\hline Double electron & 12 & 8 \\
\hline Double muon & 13 & 5 \\
\hline Double tau & 29,20 & 12 \\
\hline Double gamma & 35,25 & 10 \\
\hline ET $_{\mathrm{T}}^{\text {miss }}$ & 80 & 17 \\
\hline Five jets & 55 & 8 \\
\hline
\end{tabular}

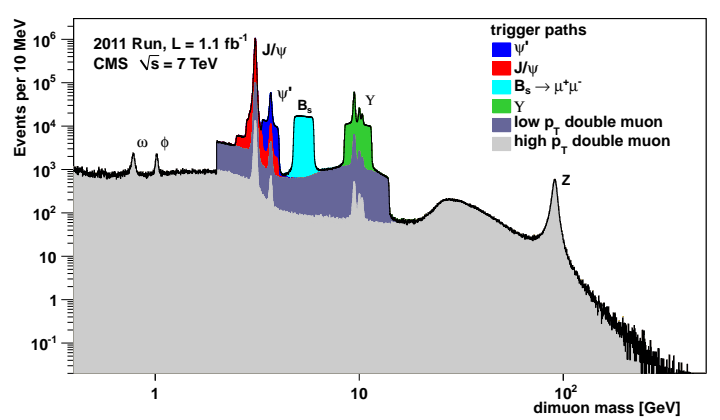

Figure 9: Left: main EF triggers in ATLAS. Right: dimuon mass spectrum as collected by CMS, with different HLT selections devoted to flavour physics shown in different colors.

\section{Conclusions}

Despite different main physics goals of ATLAS, CMS and LHCb, and differences in detector designs, similar technical challenges were faced in the trigger developments, and similar hardware and software architectures have been adopted. First level trigger systems operate according to similar principles, measuring energy deposits in the calorimeters and searching for muon candidates 
passing some minimal quality and momentum requirements. The high level trigger algorithms use similar techniques but pursue different goals. In LHCb, the trigger system aims at the most inclusive selection of beauty decays, exploiting mainly the reconstruction of vertices and tracks in the silicon detectors. In ATLAS and CMS, the trigger system must verify hundreds of different selections to cover the wide physics programme of these general purpose detectors. Heavy flavour physics is essentially limited to final states with muons, due to the huge QCD production at very high luminosities: the main triggers are dimuon triggers exploiting vertex, mass, and momentum constraints. The operation of the trigger systems has been very successful during the first run of the LHC, despite the challenges offered by the machine, which has been providing very large event pileup and luminosity. Very good selection efficiencies and high purities were achieved, while rates were kept under control. Trigger systems will undergo several upgrades, together with the detectors, to face the future improvement of the LHC performance in terms of center-of-mass energy and peak luminosity.

\section{References}

[1] LHCb Collaboration, "The LHCb Detector at the LHC” JINST 3, S08005 (2008).

[2] ATLAS Collaboration, “The ATLAS Experiment at the CERN Large Hadron Collider," JINST 3, S08003 (2008).

[3] CMS Collaboration, “The CMS experiment at the CERN LHC,” JINST 3, S08004 (2008).

[4] R Aaij et al., "The LHCb Trigger and its Performance in 2011," JINST 8, P04022 (2013)

[5] ATLAS Collaboration, "ATLAS first level trigger: Technical design report," CERN-LHCC-98-14.

[6] CMS Collaboration, "CMS. The TriDAS project. Technical design report, vol. 1: The trigger systems," CERN-LHCC-2000-038.

[7] CMS Collaboration, "CMS, the Compact Muon Solenoid. Muon technical design report," CERN-LHCC-97-32.

[8] ATLAS Collaboration, "ATLAS muon spectrometer: Technical design report," CERN-LHCC-97-22.

[9] CMS Collaboration, "Efficiency of the CMS level 1 muon trigger system in 2011 and 2012, CERN-CMS-DP-2012-019.

[10] V. V. Gligorov and M. Williams, "Efficient, reliable and fast high-level triggering using a bonsai boosted decision tree,” JINST 8, P02013 (2013) [arXiv:1210.6861 [physics.ins-det]].

[11] ATLAS Collaboration, "ATLAS high-level trigger, data acquisition and controls: Technical design report," CERN-LHCC-2003-022.

[12] CMS Collaboration, "CMS: The TriDAS project. Technical design report, Vol. 2: Data acquisition and high-level trigger," CERN-LHCC-2002-026.

[13] ATLAS Collaboration, "Performance of the ATLAS muon trigger in 2011', ATLAS-CONF-2012-099.

[14] CMS Collaboration, "Single Muon efficiencies in 2012 Data," CERN-CMS-DP-2013-009.

[15] CMS Collaboration, "Performance of CMS muon reconstruction in pp collision events at $\sqrt{s}=7$ TeV,’ JINST 7, P10002 (2012) [arXiv:1206.4071 [physics.ins-det]]. 